- Proc. 1lth Intern. Conf. on Positron Annihilation (ICPA-11), Kansas City, MO, May 25-30, 1997."

\title{
CONF-9705156--1
}

BNL-64583

\section{Two-Detector Doppler Broadening Profiles in Al}

\author{
P.E. Mijnarends', A.C. Kruseman', A. van Veen', V.J. Ghosh', \\ P. Asoka-Kumar', A. Bansil ${ }^{3}$, S. Kaprzyk ${ }^{4}$ and K.G. Lynn ${ }^{5}$ \\ 'IRI. Delft University of Technology, 2629 JB Delft, The Netherlands \\ 'Brookhaven National Laboratory, Upton, NY 11973 \\ 'Department of Physics, Northeasterm University, Boston MA 02115 \\ Academy of Mining and Metallurgy, 31214 Krakow, Poland \\ ${ }^{5}$ Materials Research Center, Washington State Univ., Pullman, WA 99163
}

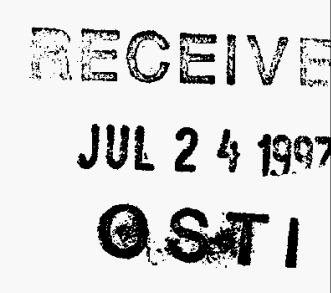

Keywords: positrons, Doppler broadening, lifetime, dual detector, electronic structure. KKR, aluminium

\begin{abstract}
Low-background Doppler broadening profiles have been measured in Al. The KKR methodology has been used to calculate the profile and study the effect of positron-electron correlation.

\section{Introduction}

The two-detector Doppler broadening method [1] attracts considerable interest as a technique for the detection, study and characterization of (defects in) materials. A measurement of the annihilation radiation of positron-electron $\left(\mathrm{e}^{+}-\mathrm{e}^{-}\right)$pairs with the aid of two collinear solid state detectors in coincidence yields the Doppler-broadened annihilation lineshape with a strongly suppressed background and improved energy resolution. This facilitates the study of annihilations with core electrons. particularly of atoms neighbouring a vacancy, and leads to increased elemental specificity of positron annihilation spectroscopy [2.3]. It also opens the possibility to study $\mathrm{e}^{+}-\mathrm{e}^{-}$correlation over a greatly extended range of momenta.

Calculations of the high-momentum part of Doppler broadening curves have so far been based predominantly on the overlapping free-atom model [4]. This yields useful results in the highmomentum regime where core annihilation dominates, but is unsatisfactory at low momenta where most annihilations involve valence electrons and band-structure effects are important. Furthermore. the valence electrons also affect the high-momentum part of the curve directly by superimposing high-momentum components (HMC) of their wavefunctions on the core contribution, and indirectly through their effect on the $e^{*}$ wavefunction, particularly when vacancies are present. Thus, other methods which produce full crystal wavefunctions and describe the full momentum range more satisfactorily should be explored. Comparison between experiment and theory should then give insight in $\mathrm{e}^{+}-\mathrm{e}^{-}$correlation. In the present study of Al we have employed the KKR-based methodology used successfully in high- $T_{\mathrm{c}}$ and other materials [5].
\end{abstract}

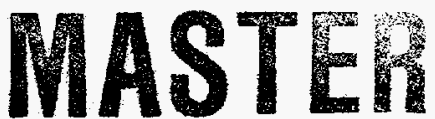

\section{Theory}

The electronic structure of core and valence electrons for $\mathrm{Al}(a=0.4050 \mathrm{~nm})$ has been calculated self-consistently at $489 k$ points in 1/48th of the Brillouin zone [6]. Valence electron wavefunctions include spherical waves up to and including $\ell=3$. The positron crystal potential contains a $\mathrm{e}^{+}-\mathrm{e}^{-}$ correlation term obtained via parametrization [7] of the results of Arponen and Pajanne [8]. The same band-structure methods as used for the electrons are applied to generate the positron wavefunction. The momentum density $\rho^{2 \gamma}(p)$ of the annihilating positron-electron pairs is given by

$$
\left.\rho^{2 \gamma}(p)=\pi r_{e}^{2} c \sum_{j} \| d r \exp (-i p . r) \psi_{j}(r) \phi_{+}(r) \vee \gamma[n .(r)]\right]^{2},
$$


where $\gamma[n .(r)]$ represents the enhancement of the annihilation probability due to $\mathrm{e}^{+}-\mathrm{e}^{-}$correlations, $n .(r)$ denotes the electron density, and the other symbols have their usual meaning. The integration is carried out by expanding $\gamma$ into plane waves and convoluting it with the plane-wave expansion of the positron wavefunction. The result is then convoluted with the plane-wave expansions of the electronic wavefunctions [5]. $\rho^{2 \gamma}(p)$ is calculated at $-5 \times 10^{7} p$ points filling a cube in $p$ space of side 230 in units of $10^{-3} m_{0} c$, with $m_{0}$ the electronic rest mass and $c$ the velocity of light (a momentum of $10^{-3} m_{0} c$ corresponds to an angle of $1 \mathrm{mrad}$ in an angular correlation experiment). All calculations are well converged and corrected for resolution. The Doppler profile is obtained by integration of $\rho^{2 \gamma}(p)$ with respect to two of the three momentum components, while the positron lifetime is obtained by integrating $\rho^{2 \gamma}(p)$ over all momenta.

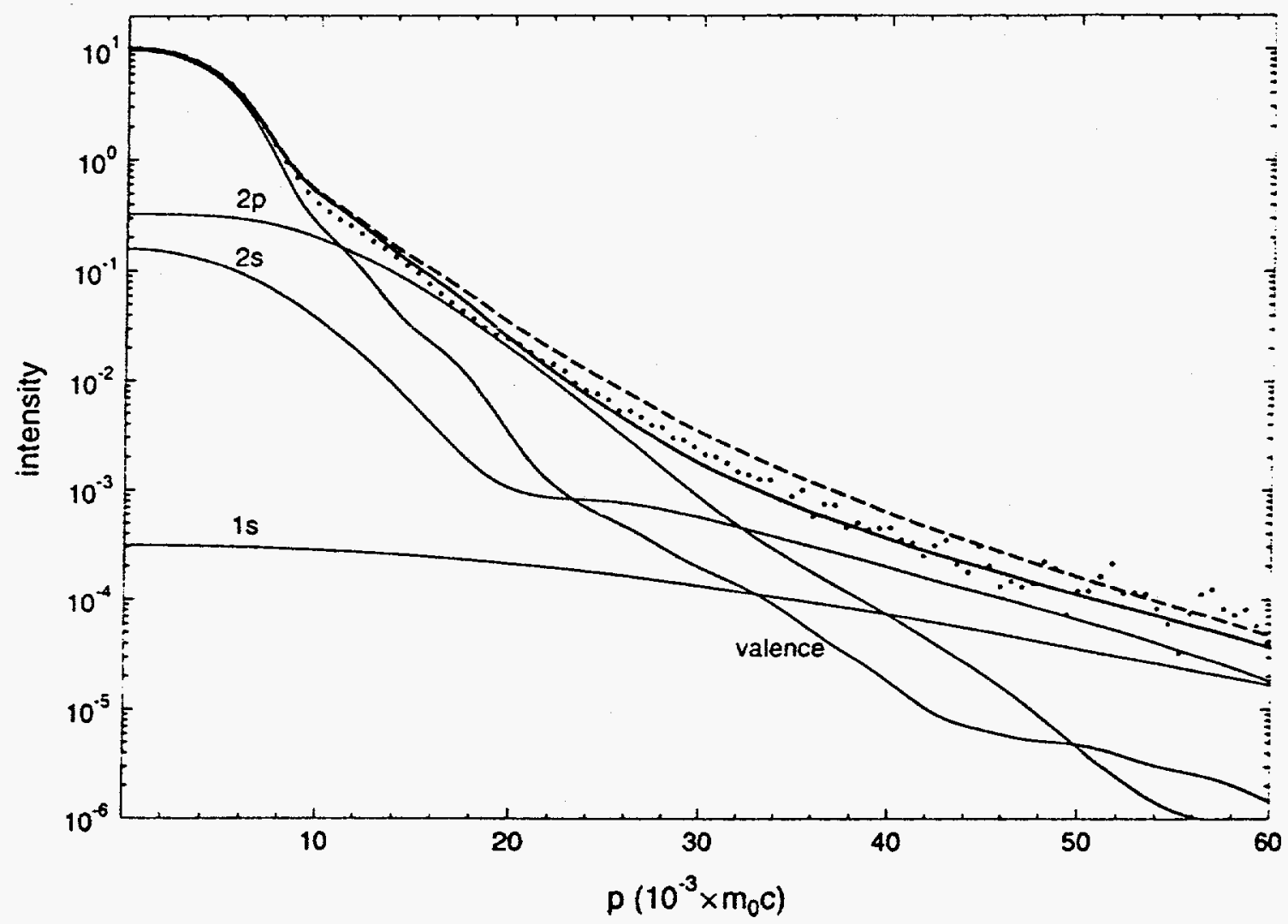

Fig. I Doppler broadening curves in Al [100] for LDA [9] (solid) and GGA [11] (dashed) enhancement. The LDA core and valence contributions are shown separately. Theory and experiment have been normalized at $p=0$.

\section{Results and discussion}

Figure 1 shows experimental results for the Doppler broadening of the annihilation radiation emitted along the [100] direction in an Al single crystal. measured with two Ge detectors in coincidence (total resolution $0.90 \mathrm{keV}$ FWHM) at the beam at Delft. These data are consistent with similar results obtained at Brookhaven with a lower resolution. They are compared with the results of three KKR calculations. In the first one, enha irement has been applied according to the local density approximation (LDA), i.e., $\gamma_{L D A}(r)=g_{0}\left(r_{s}\right)$ of Ref. [9]. The agreement with experiment is fair over the entire range of momenta except between 10 and $20 \times 10^{-3} m_{10} c$ where the valence electron HMC's contribute to the fact that the calculated density is slightly higher than the experimental one. 
Because of the large solid angles subtended by the detectors. the direction of the resolved momentum component is not well defined in the experiment: this may well result in the measured HMC's being smaller than expected. The $\mathrm{e}^{+}$lifetime $\tau$ is found to be $166 \mathrm{ps}$, in fairly good agreement with the experimental value of 163 ps [10]. The second calculation (not shown in Fig. 1) employs the stronger enhancement introduced by Barbiellini et al. [11]. It leads to a slightly worse fit (between 20 and $35 \times 10^{-3} \mathrm{~m}_{0} \mathrm{c}$ ) and a lifetime $\tau=143 \mathrm{ps}$ which is far too short.

The third calculation employs $\mathrm{e}^{+}-\mathrm{e}^{-}$correlation and enhancement according to the generalized gradient approximation (GGA) [11]. This yields $\tau=161$ ps (significantly longer than found earlier [11]) but the strong non-linearity of $\gamma_{G G A}(r)$ introduces a considerable amount of high momentum. Consequently, more plane waves are required to obtain convergence and the $2 s$ and $2 p$ contributions to the profile become broader, resulting in significant disagreement with the experimental data (up to a factor of 1.5 ) between -10 and $-50 \times 10^{-3} m_{0} c$.

Although it would be premature to draw general conclusions concerning $\mathrm{e}^{+}-\mathrm{e}^{-}$correlation from this work before a range of representative perfect and defected metals have been considered, the present results seem to favour an enhancement factor which is a weaker function of the electron density than the factors used in the second and third calculation [11]. In general, however, wellconverged calculations of the electronic structure and the momentum density are essential before conclusions can be drawn concerning $\mathrm{e}^{+}-\mathrm{e}^{-}$correlation.

\section{Acknowledgments}

This work is sponsored by the National Computer Facilities Foundation (NCF) for the use of supercomputer facilities with financial support from the Netherlands Organization for Scientific Research (NWO). It is also supported by the US Department of Energy under contract W-31-109-ENG-38. including a subcontract to Northeastern University, and benefited from the allocation of supercomputer time at NERSC and at the Pittsburgh Supercomputer Center. One of the authors (VJG) was supported in part by the U.S. Department of Energy under contract No. DE-AC02-76CH00016. We are grateful for travel grants from NATO. NWO. KNAW and Shell Travel Services.

\section{References}

[1] K.G. Lynn. J.R. MacDonald, R.A. Boie, L.C. Feldman. J.D. Gabbe, M.F. Robbins, E. Bonderup and J. Golovchenko. Phys. Rev. Lett. 38 (1977), p. 241.

[2] P. Asoka-Kumar. M. Alatalo, V.J. Ghosh, A.C. Kruseman, B. Nielsen and K.G. Lynn, Phys. Rev. Lett. 77 (1996), p. 2097.

[3] A.C. Kruseman. H. Schut. A. van Veen. P.E. Mijnarends, M. Clement and J.M.M. de Nijs, Appl. Surf. Sci. (in press).

[4] M. Alatalo. B. Barbiellini, M. Hakala. H. Kauppinen, T. Korhonen. M.J. Puska. K. Saarinen, P. Hautojärvi and R.M. Nieminen. Phys. Rev. B54 (1996), p. 2397: M. Alatalo. P. AsokaKumar, V.J. Ghosh, B. Nielsen, K.G. Lynn, A.C. Kruseman, A. van Veen, T. Korhonen and M.J. Puska. J. Phys. Chem. Solids (in press).

[5] P.E. Mijnarends and A. Bansil, in: "Positron Spectroscopy of Solids", edited by A. Dupasquier and A.P. Mills jr., IOS Press, Amsterdam 1995, p. 25.

[6] S. Kaprzyk and A. Bansil. Phys. Rev. B 42 (1990) p.7358; A. Bansil and S. Kaprzyk, Phys. Rev. B 43 (1991) p. 10335; A. Bansil, S. Kaprzyk and J. Tobola. MRS Symp. Proc. 253 (1992), p. 505.

[7] E. Boroński and R.M. Nieminen, Phys. Rev. B34 (1986), p. 3820.

[8] J. Arponen and E. Pajanne, Ann. Phys. (NY) 121 (1979), p. 343.

[9] M.J. Puska, A.P. Seitsonen and R.M. Nieminen. Phys. Rev. B52 (1995), p. 1094.

[10] A. Seeger, F. Barnhart and W. Bauer, in: "Positron Annihilation", edited by L. DorikensVanpraet, M. Dorikens and D. Segers (World Scientific, Singapore 1989), p. 275.

[11] B. Barbiellini. M.J. Puska, T. Torsti and R.M. Nieminen, Phys. Rev. B51 (1995), p. 7341. 


\section{DISCLAIMER}

This report was prepared as an account of work sponsored by an agency of the United States Government. Neither the United States Government nor any agency thereof, nor any of their employees, make any warranty, express or implied, or assumes any legal liability or responsibility for the accuracy, completeness, or usefulness of any information, apparatus, product, or process disclosed, or represents that its use would not infringe privately owned rights. Reference herein to any specific commercial product, process, or service by trade name, trademark, manufacturer, or otherwise does not necessarily constitute or imply its endorsement, recommendation, or favoring by the United States Government or any agency thereof. The views and opinions of authors expressed herein do not necessarily state or reflect those of the United States Government or any agency thereof. 


\section{DISCLAIMTER}

Portions of this document may be illegible in electronic image products. Images are produced from the best available original document. 